\title{
Comparison of ultrasound therapy and radial shock wave therapy in the treatment of venous leg ulcers - clinical, pilot study
}

\author{
Patrycja Dolibog ${ }^{1}$, Paweł T. Dolibog ${ }^{1}$, Andrzej Franek ${ }^{1}$, Ligia Brzezińska-Wcisło², Beata Wróbel' ${ }^{1}$, Hubert Arasiewicz ${ }^{2}$, \\ Daria Chmielewska ${ }^{3}$, Jacek Ziaja ${ }^{4}$, Edward Błaszczak ${ }^{1}$
}

${ }^{1}$ Chair and Department of Medical Biophysics, School of Medicine, Medical University of Silesia, Katowice, Poland ${ }^{2}$ Department of Dermatology, School of Medicine, Medical University of Silesia, Katowice, Poland

${ }^{3}$ Department of Basics of Physiotherapy, Faculty of Physiotherapy, Academy of Physical Education, Katowice, Poland

${ }^{4}$ Department of General, Vascular and Transplant Surgery, School of Medicine, Medical University of Silesia, Katowice, Poland

Adv Dermatol Allergol 2018; XXXV (5): 454-461

DOI: https://doi.org/10.5114/ada.2018.79191

\begin{abstract}
Introduction: Venous insufficiency and venous leg ulcers each year affect more and more people, especially in developed countries. The challenge of our time is to find an effective method of treating venous leg ulcers, which will further shorten treatment time and reduce the cost of treatment.

Aim: To compare the effects of treating venous leg ulcers using ultrasound therapy, radial shock wave therapy and standard care.

Material and methods: Group A consisted of 17 patients. Patients were treated with ultrasound therapy US power density $0.5 \mathrm{~W} / \mathrm{cm}^{2}$, pulsed wave with a duty cycle of $1 / 5$, and $1 \mathrm{MHz}$ frequency. Group B consisted of 17 patients. Patients were treated with the radial shock wave R-ESWT using surface energy density $0.17 \mathrm{~mJ} / \mathrm{mm}^{2}, 100 \mathrm{impulses} / \mathrm{cm}^{2}$, frequency of $5 \mathrm{~Hz}$ and a pressure of $0.2 \mathrm{MPa}$. Group C (control group) consisted of 17 patients. Patients in this group received standard care: gauze dressing saturated in $0.9 \%$ sodium chloride and elastic bandages changed daily for 4 weeks.

Results: Ultrasound therapy with $1 \mathrm{MHz}$ and energy power density $0.5 \mathrm{~W} / \mathrm{cm}^{2}$ for 4 weeks resulted in an average reduction of $68 \%$ of the area of ulceration. We used for venous leg ulcers 4-week treatment with radial shock wave therapy resulting in a $38 \%$ mean percentage reduction of the ulceration area. Standard care reduces the area of ulceration by only $16 \%$.

Conclusions: The use of ultrasound therapy for the treatment of venous leg ulcers is more effective than the use of radial shock wave therapy or standard care alone.
\end{abstract}

Key words: leg ulcers, ultrasound therapy, radial shockwave.

\section{Introduction}

Health problems related to venous leg ulcer affect many people, and the challenge is to find an effective treatment method for venous ulcers, in consideration of increasing healing costs [1].

The aim of the healing process and the condition of effective healing of the ulceration of the lower leg is to lower the venous pressure and keep it in the lower limb vessels. Compression therapy, as the primary method to assist the healing of venous leg ulcers, consists in exerting gradual compression on the affected limb. The use of adequate external force reduces the local blood volume by redistributing it in the proximal direction, thereby lowering the hydrostatic pressure in the limb and restoring proper rheological conditions. Blood stasis is reduced, resulting in a faster flow of white blood cells, which reduces the adhesion and endothelial injury. Increased venous flow does not affect arterial flow in any way [1].

Physiotherapy has continued to grow in recent years, with a growing number of new types of medical devices, and technical solutions enable their applications. Physical methods of treating venous leg ulcers include low

Address for correspondence: Patrycja Dolibog PhD, Chair and Department of Medical Biophysics, School of Medicine, Medical University of Silesia, 18 Medyków St, 40-752 Katowice, Poland, phone: +48 602762 442, e-mail: pdolibog@sum.edu.pl Received: 16.10.2017, accepted: 16.11.2017. 
frequency magnetic therapy, high voltage electric stimulation and direct current stimulation, laser biostimulation, ultrasound (US) therapy and extracorporeal shock wave therapy (ESWT). The last two interact mechanically on the tissue, changing the pressure and compressing it [2-10].

Ultrasound therapy has been used in wound healing for over 20 years while ESWT in wound treatment has been applied only for the last several years. Both physical methods are methods using mechanical waves, so it is worth comparing their effectiveness. There are many methods of applying ultrasound and shock wave therapy available in clinical trials, but there is no clear answer as to what values are most effective in everyday practice [2-10].

\section{Aim}

The aim of our study was to compare the effects of treating venous leg ulcers using ultrasound therapy, radial shock wave therapy and a control group. The study assesses the influence of physical therapy on reduction ulcer surface area, Gilman index and comparisons of measured indicators between all groups.

\section{Material and methods}

\section{Design and criteria}

The study was conducted among 51 adults (over 18 years) with venous leg ulcers (29 females and 22 males) who agreed to participate in the study and signed written consent forms. The randomized clinical study was conducted in the Department of Dermatology at the Medical University of Silesia. The project, methodology and study were approved by the local Bioethical Committee of Medical University Silesia (protocol number KNW/0022/KB1/25/II/15).

A total of 51 patients were qualified for the therapy and randomly assigned to three comparison groups in a one-to-one ratio into the ultrasound therapy group, the radial shockwave therapy group and the control group.

\section{Procedures}

All patients qualify for the study were diagnosed with venous ulcer, had an ankle brachial pressure index (ABPI) $>1$, and underwent standard blood morphology and urine testing. The exclusion criteria were: diabetes, cancer, rheumatoid arthritis, ventricular arrhythmia, cardiac pacemaker, ulcer surgery, skin infection, steroid therapy, bilateral ulcers, lymphedema, congestive heart failure.

All ulcers were diagnosed according to venous ultrasound examination of arteries and veins of lower limbs performed using an Aloka Prosound Alpha 6 ultrasound device (Hitachi Aloka Medical, Ltd., Japan). Doppler examination was performed on both legs (the study blood flow in iliac, proximal and distal segments of femoral, popliteal, upper and lower segments of great saphenous, and small saphenous veins).

All patients in all groups were treated by dermatologists (study design, methodology and treatment coordination), vascular surgeons (ultrasound examination of arteries and veins of lower limbs, clinical, etiologic, anatomic, pathologic classification), physiotherapists (ultrasound and shock wave therapy) and nurses (standard care, wound area measurements, data collections).

The healing rates were assessed on the basis of wound healing rates using digital planimetry (Kurta XGT, Altek Inc, USA) and systematically photographed. The digital image of the wound was measured using computer software (C-GEO v. 4.0, Nadowski, Poland). Total ulcer area $\left(\mathrm{cm}^{2}\right)$, max length $(\mathrm{cm})$ and max width $(\mathrm{cm})$ of the wound were measured. Wounds were measured before treatment, 14 days after the start of treatment and after each treatment.

The healing process between groups were assessed using percentage changes of total ulcer area: $\Delta S \%=\left(\left(S_{1}\right.\right.$ $\left.\left.-S_{\mathrm{F}}\right) \times 100 \%\right) / S_{1}$, where $\Delta S \%$ - relative change of ulcer surface area (\%), $S_{1}, S_{F}$ - initial and final ulcer area $\left(\mathrm{cm}^{2}\right)$.

The Gilman index is a linear parameter for assessing wound healing in two dimensions. Determining the average distance that the edge of the wound was healed during treatment towards the center of the wound: $d=$ $\left(2\left(S_{F}-S_{1}\right)\right) /\left(C_{F}+C_{1}\right)$, where $d-$ Gilman index $(\mathrm{cm}), S_{1}, S_{F}$ - initial and final ulcer area $\left(\mathrm{cm}^{2}\right), C_{1}, C_{F}$ - initial and final ulcer circumference $(\mathrm{cm})$.

\section{Patients}

Group A consisted of 17 patients. Patients were treated with ultrasound therapy US (producer: Physiomed Electromedizin AG, Germany; model: Ionoson, applicator $50 \mathrm{~mm}$ ). Treatment parameters were: power density $0.5 \mathrm{~W} / \mathrm{cm}^{2}$, pulsed wave with a duty cycle of $1 / 5$, and $1 \mathrm{MHz}$ frequency. The duration of single treatment was dependent on the ulcer size $\left(1 \mathrm{~min}\right.$ for each $1 \mathrm{~cm}^{2}$ ulcer area). The procedures were repeated once daily, 5 days a week for 4 weeks. All patients in group A used standard care: gauze dressing saturated in 0.9\% sodium chloride and elastic bandages changed daily.

Group B consisted of 17 patients. Patients were treated with the radial shock wave R-ESWT (producer: Gymna Uniphy; model: ShockMaster 500; applicator: classic $15 \mathrm{~mm}$ ). Treatment parameters were: surface energy density $0.17 \mathrm{~mJ} / \mathrm{mm}^{2}, 100 \mathrm{impulses} / \mathrm{cm}^{2}$, frequency of $5 \mathrm{~Hz}$ and a pressure of $0.2 \mathrm{MPa}$. The R-ESWT was provided six times at intervals of 5 days for 4 weeks. All patients in group B used standard care: gauze dressing saturated in $0.9 \%$ sodium chloride and elastic bandages changed daily.

The treatment in groups A and B was performed using wound sterile ultrasound transmission gel (Aquasonic 100) then a sterile operation foil was glued (elastoFILM Company Outline, Poland), on which the re-applied gel for ultrasound transmission was placed. 
Group C (control group) consisted of 17 patients. Patients in this group received standard care: gauze dressing saturated in $0.9 \%$ sodium chloride and elastic bandages changed daily for 4 weeks.

Patients in all comparison groups were homogeneous in terms of basic characteristics, i.e. age, gender, body mass index, smokers, body height (Table 1), and also homogeneous as regards the initial measurements wound size, duration time of ulcer and ulcer location (Table 2).

\section{Data collection}

Measured values were entered into the Excel (Microsoft Office Excel 2013, USA) database and coded, then sent to a statistical coordinator from the Department of Biophysics.

\section{Statistical analysis}

Statistical analysis was performed using the Statistica program (StatSoft version 12, Poland). Data are shown as mean and standard deviation for quantitative data or number and percentage for qualitative data. Initially, all results obtained from patients were compared using the Shapiro-Wilk test. The prevalence of features in the groups was compared with $\chi^{2}$ independence test (greatest reliability level). Subsequently, the Kruskal-Wallis two-way test was used to compare

Table 1. Details of patients in all comparative groups

\begin{tabular}{|c|c|c|c|c|}
\hline Parameter & $\begin{array}{c}\text { Ultrasound therapy } \\
\text { Group A }\end{array}$ & $\begin{array}{l}\text { Radial ESWT } \\
\text { Group B }\end{array}$ & $\begin{array}{l}\text { Control } \\
\text { Group C }\end{array}$ & $P$-value \\
\hline Number of patients & 17 & 17 & 17 & \\
\hline \multicolumn{5}{|l|}{ Gender, $n(\%)$ : } \\
\hline Female & $8(47)$ & $8(47)$ & $13(76)$ & $>0.05^{*}$ \\
\hline Male & $9(53)$ & $9(53)$ & $4(25)$ & \\
\hline \multicolumn{5}{|l|}{ Smokers, $n(\%)$ : } \\
\hline Yes & $14(82)$ & $13(76)$ & $15(88)$ & $>0.05^{*}$ \\
\hline No & $3(18)$ & $4(24)$ & $2(12)$ & \\
\hline \multicolumn{5}{|c|}{ Adiposity (body mass index) $\left[\mathrm{kg} / \mathrm{m}^{2}\right], n(\%)$ : } \\
\hline$n<30$ & $10(59)$ & $12(71)$ & $14(82)$ & $>0.05^{*}$ \\
\hline$n \geq 30$ & $7(41)$ & $5(29)$ & $3(18)$ & \\
\hline Age [years]: & & & & $>0.05^{\star *}$ \\
\hline Range & $47-82$ & $62-88$ & $44-88$ & \\
\hline Average & 67.7 & 71.1 & 64.4 & \\
\hline Median & 69 & 67.0 & 60.0 & \\
\hline SD & 9.2 & 9.5 & 12.5 & \\
\hline Height $[\mathrm{cm}]:$ & & & & $>0.05^{\star *}$ \\
\hline Range & $158-175$ & $158-176$ & $156-175$ & \\
\hline Average & 165.5 & 168.2 & 163.6 & \\
\hline Median & 163 & 168 & 164.0 & \\
\hline SD & 8.0 & 7.3 & 4.3 & \\
\hline Weight [kg]: & & & & $<0.05^{* x}$ \\
\hline Range & $60-110$ & $65-110$ & $49-110$ & \\
\hline Average & 82.3 & 81.3 & 67.6 & \\
\hline Median & 79.0 & 82.5 & 63.0 & \\
\hline SD & 16.9 & 10.7 & 16.1 & \\
\hline
\end{tabular}

${ }^{*} \chi^{2}$ test; ${ }^{* *}$ Kruskal-Wallis ANOVA rank test. 
Table 2. Baseline characteristics of ulcers in all comparative groups

\begin{tabular}{|c|c|c|c|c|}
\hline Parameter & $\begin{array}{l}\text { Ultrasound therapy } \\
\text { Group A }\end{array}$ & $\begin{array}{l}\text { Radial ESWT } \\
\text { Group B }\end{array}$ & $\begin{array}{l}\text { Control } \\
\text { Group C }\end{array}$ & $P$-value \\
\hline Number of patients & 17 & 17 & 17 & \\
\hline Duration of ulcer [months]: & & & & $>0.05^{* *}$ \\
\hline Range & $1-24$ & $3-24$ & $1-31$ & \\
\hline Average & 5.3 & 8.1 & 8.8 & \\
\hline Median & 3 & 5 & 9 & \\
\hline SD & 5.9 & 6.5 & 7 & \\
\hline Initial wound area $\left[\mathrm{cm}^{2}\right]$ : & & & & $>0.05^{* *}$ \\
\hline Range & $0.3-44.9$ & $1.4-47.1$ & $1.9-33.5$ & \\
\hline Average & 9.8 & 9.2 & 11.8 & \\
\hline Median & 4.6 & 4.6 & 11.2 & \\
\hline SD & 12.5 & 11.1 & 7.5 & \\
\hline Initial wound circuit [cm]: & & & & $>0.05^{\star *}$ \\
\hline Range & $2.4-26.8$ & $4.7-27.6$ & $5.1-35.8$ & \\
\hline Average & 10.8 & 11.5 & 13.9 & \\
\hline Median & 7.8 & 8.8 & 13.5 & \\
\hline SD & 7.0 & 6.2 & 7.2 & \\
\hline Initial wound length $[\mathrm{cm}]$ : & & & & $>0.05^{* *}$ \\
\hline Range & $1.0-10.5$ & $1.6-9.2$ & $1.9-14.5$ & \\
\hline Average & 4.2 & 4.0 & 5.4 & \\
\hline Median & 3.1 & 3.2 & 4.8 & \\
\hline SD & 2.8 & 2.1 & 3.0 & \\
\hline Initial wound width $[\mathrm{cm}]$ : & & & & $>0.05^{\star *}$ \\
\hline Range & $0.5-6.3$ & $0.9-7.1$ & $1.3-7.8$ & \\
\hline Average & 2.6 & 2.6 & 3.3 & \\
\hline Median & 1.9 & 2.1 & 3.2 & \\
\hline SD & 1.6 & 1.5 & 1.5 & \\
\hline Localization of VLU: & & & & $>0.05^{*}$ \\
\hline Above medial malleoli & 7 & 5 & 6 & \\
\hline Above lateral malleoli & 5 & 8 & 4 & \\
\hline Anterior shin & 2 & 1 & 3 & \\
\hline Posterior shin & 3 & 3 & 4 & \\
\hline
\end{tabular}

${ }^{*} \chi^{2}$ test; ${ }^{* *}$ Kruskal-Wallis ANOVA rank test.

the quantitative variables in all comparative groups. Changes of values inside groups were performed using the Wilcoxon test. The significance level was set at $p<0.05$.

\section{Results}

Group A (ultrasound therapy) consisted of 17 patients, including 8 women and 9 men. The average age of the patients was $67.7 \pm 9.2$ years; the duration of the ulcers ranged from 1 to 24 months. In this group 18\% of the respondents were smokers, and $82 \%$ of people declared that they did not smoke. Furthermore, in this group $41 \%$ of the patients were obese.

In B group (radial shock wave) there were 17 patients, including 8 women and 9 men. The average age of the patients was $62.0 \pm 9.5$ years; the duration of the ulcers ranged from 3 to 24 months. In this group $24 \%$ of the respondents were smokers, and $76 \%$ of people declared that they did not smoke. Furthermore, in this group 29\% of the patients were obese.

Group C (control group) consisted of 17 patients, including 13 women and 4 men. The average age of the patients was $64.4 \pm 12.5$ years; the duration of the ulcers 
Table 3. Change of the value of ulcers in all groups between baseline and week 4

\begin{tabular}{|c|c|c|c|c|c|}
\hline \multirow[t]{2}{*}{ Parameter } & \multirow{2}{*}{\multicolumn{2}{|c|}{ Group }} & \multicolumn{2}{|c|}{ Mean \pm SD } & \multirow[t]{2}{*}{$P$-value } \\
\hline & & & Before therapy & After therapy & \\
\hline \multirow{3}{*}{$\begin{array}{l}\text { Total ulcer } \\
\text { surface area } \\
{\left[\mathrm{cm}^{2}\right]}\end{array}$} & A & Ultrasound therapy & $9.8 \pm 12.5$ & $5.6 \pm 9.0$ & $<0.05^{*}$ \\
\hline & B & Radial shock wave therapy & $9.3 \pm 11.1$ & $6.9 \pm 10.0$ & $<0.05^{\star}$ \\
\hline & C & Control group & $11.8 \pm 7.5$ & $10.1 \pm 6.3$ & $<0.05^{\star}$ \\
\hline \multirow[t]{3}{*}{ Circuit $[\mathrm{cm}]$} & A & Ultrasound therapy & $10.8 \pm 7.0$ & $6.9 \pm 7.5$ & $<0.05^{*}$ \\
\hline & B & Radial shock wave therapy & $11.5 \pm 6.2$ & $9.7 \pm 6.6$ & $<0.05^{*}$ \\
\hline & C & Control group & $13.9 \pm 7.2$ & $12.6 \pm 7.0$ & $<0.05^{*}$ \\
\hline \multirow[t]{3}{*}{ Length [cm] } & A & Ultrasound therapy & $4.2 \pm 2.8$ & $2.8 \pm 3.1$ & $<0.05^{*}$ \\
\hline & B & Radial shock wave therapy & $4.0 \pm 2.1$ & $3.4 \pm 2.3$ & $<0.05^{*}$ \\
\hline & C & Control group & $5.4 \pm 3.0$ & $4.9 \pm 2.9$ & $<0.05^{*}$ \\
\hline \multirow[t]{3}{*}{ Width [cm] } & A & Ultrasound therapy & $2.6 \pm 1.6$ & $1.4 \pm 1.6$ & $<0.05^{*}$ \\
\hline & B & Radial shock wave therapy & $2.6 \pm 1.5$ & $2.3 \pm 1.8$ & $>0.05^{*}$ \\
\hline & C & Control group & $3.3 \pm 1.5$ & $2.9 \pm 1.5$ & $<0.05^{*}$ \\
\hline
\end{tabular}

*Wilcoxon test.

ranged from 3 to 31 months. In this group $12 \%$ of the respondents were smokers, and $88 \%$ of people declared that they did not smoke. Furthermore, in this group 18\% of the patients were obese.

All participants completed the entire study program. After completion of the therapy, all the comparison groups demonstrated a reduction of wound size as compared to the initial values (Table 3 ). The percentage wound area reduction between baseline and the $4^{\text {th }}$ week is shown in Figure 1. However, the analysis demonstrated that the largest reduction of wound size was recorded in group A (ultrasound stimulation US), followed by group

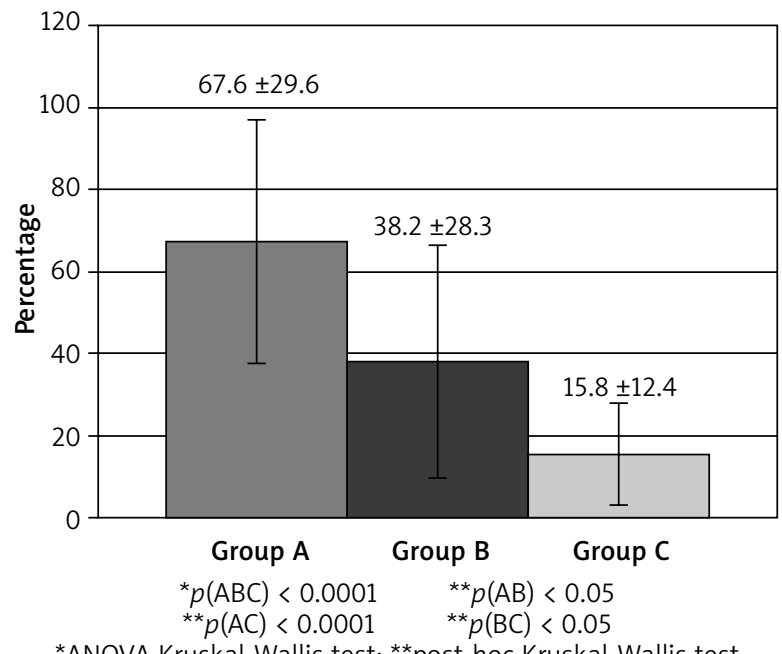

${ }^{*}$ ANOVA Kruskal-Wallis test; ${ }^{* *}$ post-hoc Kruskal-Wallis test

Figure 1. Changes of the percentage value of ulcer area in comparison between groups
$B$ (radial shock wave therapy R-ESWT), and the smallest was in group C (control group).

The analysis showed that all treatment methods used in the study proved to be effective. Comparison before the study and after 4 weeks of treatment shows a statistically significant decrease in total ulcer area in all groups.

Ultrasound therapy with $1 \mathrm{MHz}$ and energy power density $0.5 \mathrm{~W} / \mathrm{cm}^{2}$ for 4 weeks resulted in an average reduction of $67.6 \pm 29.6 \%$ of the area of ulceration. We used for 4 weeks venous leg ulcer treatment with radial shock wave therapy resulting in a $38.2 \pm 28.3 \%$ mean percentage reduction of ulceration area. Standard care reduces the area of ulceration by only $15.8 \pm 12.4 \%$.

Analysis with the Gilman index showed that the largest effects of treatment were in the ultrasound therapy (group A) and radial shock wave therapy (group B) groups compared with the control group (group C). The Gilman index was $0.49 \pm 0.20 \mathrm{~cm}$ for patients who received ultrasound therapy; for patients using radial shock wave it was $0.24 \pm 0.17 \mathrm{~cm}$ and for patients who used standard care alone it was only $0.13 \pm 0.01 \mathrm{~cm}$.

The comparison between groups in terms of the Gilman index is shown in Figure 2.

\section{Discussion}

The use and comparison of the results of the treatment of venous leg ulcers using ultrasound and shock wave has not yet been recorded. In this aspect, our work is new.

Escandon et al. [2] assessed the effectiveness of noncontact low-frequency ultrasound (NLFU) treatment ve- 
nous leg ulcers (9 patients). Patients were treated 3 times per week for 4 weeks. Minimum treatment time was 3 min for wound area $<10 \mathrm{~cm}^{2}$ and increased 1 min for each $10 \mathrm{~cm}^{2}$. The authors noted a statistically significant mean reduction of $45 \%$ in ulcer area and the decreases in inflammatory cytokine expression were correlated despite a non-significant decrease in bacterial count.

Gibbons et al. [3] used NLFU (40 kHz) treatment for 41 patients who had venous leg ulcers. Participants randomized to the study received the treatment 3 times per week for 4 weeks (12 treatments). Therapy duration started at 3 min for wound areas $>10 \mathrm{~cm}^{2}$ and was extended by $1 \mathrm{~min}$ for each successive $10 \mathrm{~cm}^{2}$ area. Moreover, standard care (SC) consisting of compression, moist wound dressing and debridement was used. After 4 weeks of study treatment $61.6 \pm 28.9 \%$ area reduction was reported, whereas for patients who used only standard care (40 patients) the percent area reduction was $45.0 \pm 32.5 \%$.

Olyale et al. [4] compared the effect of high and low frequency ultrasound for venous leg ulcer treatment. High frequency $(1-3 \mathrm{MHz})$ ultrasound therapy $(0.5-1 \mathrm{~W} /$ $\mathrm{cm}^{2}$ ) was applied for 5-10 min to the skin surrounding the ulcers; treatment time in minutes equaled the ulcer area in $\mathrm{cm}^{2}$. NCLFU $\left(40 \mathrm{kHz}, 0.1-0.8 \mathrm{~W} / \mathrm{cm}^{2}\right)$ treatment time was in the range from 3-20 min and similarly equaled the ulcer area in $\mathrm{cm}^{2}$. In addition, the results of treatment were compared to a group of patients who used only standard care. The ulcer area was reduced by $55.42 \%$ in the standard care alone group, by $67.25 \%$ in the additional high frequency ultrasound treatment group, and by $72.83 \%$ in the noncontact low frequency ultrasound (NCLFU) treatment group.

Similar studies have also been conducted by Beheshti et al. [5]. Ninety patients with venous leg ulcers were divided equally into three groups. In the first group only standard care was used, the second group used high frequency ultrasound and in the third NCLFU was used (40 kHz; 0.1-0.8 W/cm²). All patients received compression therapy as standard care. After 4 months of treatment in the first group, the mean surface area decreased by $46.43 \%$, in the high frequency ultrasound group it decreased by $59.03 \%$ and in the NCLFU group it decreased by $63.74 \%$.

The NCLFU therapy for hard-to-heal venous leg ulcers was used by White et al. [6]. In the first group (19 patients) only standard care was used, and in the second group (17 patients) NCLFU treatment and standard care were used. After 8 weeks of treatment patients treated with standard care showed a 39.2\% mean change in wound area, while patients treated with NCLFU treatment and standard care showed a $46.6 \%$ mean change. The difference between compared groups was not significant.

Taradaj et al. [7] used high voltage stimulation (HVS), ultrasound (US) $\left(0.5 \mathrm{~W} / \mathrm{cm}^{2} ; 1 \mathrm{MHz}\right)$, low level laser therapy (LLLT) and compression therapy (CT) for treatment

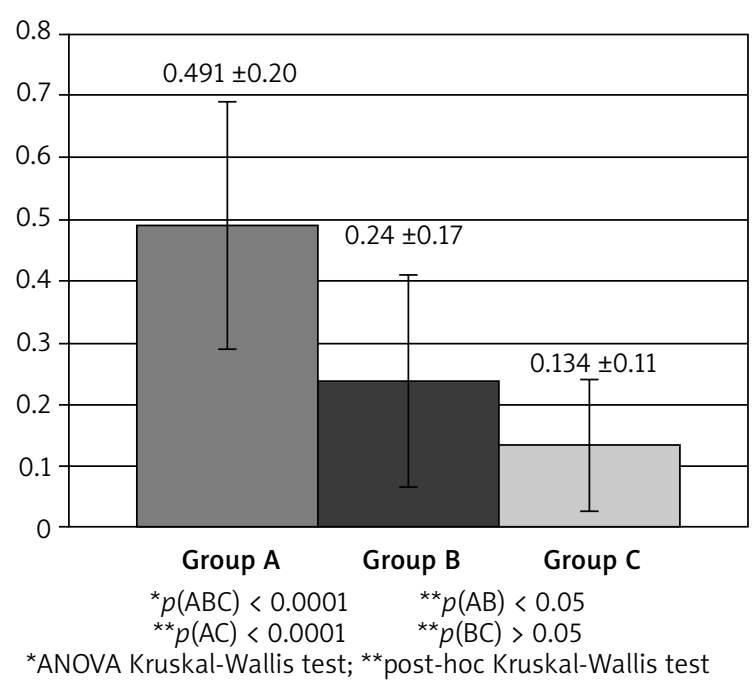

Figure 2. Comparison between groups in terms of Gilman index $[\mathrm{cm}]$

of patient with venous leg ulcers and compared it with standard care. The authors subdivided each group according to whether patients were subjected to early surgical treatment or not. Ultrasound treatments were performed daily for 7 weeks. Average change of total surface area after 7 weeks was $60.01 \%$ for patients after surgery and $56.67 \%$ for patients without surgery. The largest change was reported in post-operative patients who underwent compression therapy (78.19\%) (stocking providing 25 to $32 \mathrm{~mm} \mathrm{Hg}$ of pressure at the ankle worn for 10 to $12 \mathrm{~h}$ and removed at night).

The use of shock waves for the treatment of soft tissue defects is new. Even newer is the use of shock wave in the treatment of venous ulcers. The number of publications on this topic is small. A shock wave used to treat soft tissue defects is produced in a device outside a patient's body, called extracorporeal shock wave therapy or (ESWT), or SWT for short.

Depending on how the shock wave is produced (electromagnetic, electro-hydraulic or piezoelectric), the amount of emitted energy and its diffusion into the patient's body tissue, shock wave therapy can be divided into focused extracorporeal shock wave therapy (F-ESWT) and defocused (or unfocused) extracorporeal shock wave therapy (D-ESWT). Other transducer design can deliver radial wave ESWT (R-ESWT) or flat (planar) wave ESWT (P-ESWT).

An air-driven or electromagnetically accelerated projectile strikes the transmitter forming part of the application. The applicator is in the form of a gun, terminated with a transmitter. The wave formed in this way is a radial shock wave (RSWT).

Fioramonti et al. [8] used the ESWT shock wave to treat 2 venous ulcers in a 63-year-old patient. The applied wave had a surface energy density of $0.037 \mathrm{~mJ} / \mathrm{mm}^{2}$, frequency $4 \mathrm{~Hz}$ and $100 \mathrm{impulses} / \mathrm{cm}^{2}$. On the other leg the 
ulcer was treated only with standard care. Treatment lasted until complete cure once a week. This lasted for 6 weeks. After this time, the ulcer treated with only standard care had not completely healed.

Long-term venous ulceration in 1 patient using unfocused shock wave therapy was treated by Steiger et al. [9]. The authors used a shock wave with surface energy density of $0.25 \mathrm{~mJ} / \mathrm{mm}^{2}$, frequency $4 \mathrm{~Hz}$ and 2000 impuls$\mathrm{es} / \mathrm{cm}^{2}$. Treatment lasted for 30 weeks, after which the $\mathrm{ul}$ cer surface decreased from $15 \mathrm{~cm} \times 10 \mathrm{~cm}$ to $3 \mathrm{~cm} \times 3 \mathrm{~cm}$. Total healing was achieved through skin transplantation.

Other researchers (Sagginii et al. [10]) used ESWT in 30 patients with ulcers of various origins (11 venous ulcers). They applied surface energy density of $0.037 \mathrm{~mJ} /$ $\mathrm{mm}^{2}$ and $100 \mathrm{impulses} / \mathrm{cm}^{2}$ and a frequency of $4 \mathrm{~Hz}$. Shockwave treatments were applied every other week; patients had 4 to 10 treatments. Complete cure in the venous insufficiency subgroup was reported in $36 \%$ of patients (45\% reduction of ulcer area).

The use of ultrasound therapy for the treatment of venous leg ulcers is not new. A literature review shows that NLFU therapy is most commonly used. Frequency of the applied wave is $40 \mathrm{kHz}$, energy $0.1-0.8 \mathrm{~W} / \mathrm{cm}^{2}$. Less often used are high frequency $(1-3 \mathrm{MHz})$ ultrasound therapy $\left(0.5-1 \mathrm{~W} / \mathrm{cm}^{2}\right)$ or ultrasound US $\left(0.5 \mathrm{~W} / \mathrm{cm}^{2} ; 1 \mathrm{MHz}\right)$ [2-7].

In our study we used ultrasound power density $0.5 \mathrm{~W} /$ $\mathrm{cm}^{2}$, pulsed wave with a duty cycle of $1 / 5$, and $1 \mathrm{MHz}$ frequency. This is comparable to the literature reported above.

Applying shock wave in the treatment of venous leg ulcers is a new matter. A few reports describe the use of extracorporeal shockwave therapy with a surface energy density of $0.037 \mathrm{~mJ} / \mathrm{mm}^{2}$, frequency $4 \mathrm{~Hz}$ and 100 impuls$\mathrm{es} / \mathrm{cm}^{2}$. Other scientists have used other wave parameters: surface energy density of $0.25 \mathrm{~mJ} / \mathrm{mm}^{2}$, frequency $4 \mathrm{~Hz}$ and 2000 impulses/ $\mathrm{cm}^{2}$ [8-10].

We have not found any scientific report describing the use of radial shock wave R-ESWT in the treatment of venous leg ulcers.

The radial shock wave R-ESWT used by us had surface energy density $0.17 \mathrm{~mJ} / \mathrm{mm}^{2}, 100 \mathrm{impulses} / \mathrm{cm}^{2}$, frequency of $5 \mathrm{~Hz}$ and a pressure of $0.2 \mathrm{MPa}$.

Treatment duration and frequency of treatment were varied. When using ultrasound therapy, the most common treatments were performed 3 times a week (less frequently daily) for 4 to 16 weeks [2-7]. On the other hand, shock wave treatments were performed once a week or once every two weeks, and all treatments took 6 to 30 weeks [8-10].

In our study ultrasound treatment was repeated once daily, 5 days a week for 4 weeks, and shockwave therapy was provided six times at intervals of 5 days for 4 weeks.

A literature review shows that the mean percentage reduction of ulceration area was higher when noncreative low frequency ultrasound therapy was used than high frequency ultrasound therapy $[4,5,7]$.
The mean percentage reduction of the ulcer treated with noncreative low frequency ultrasound therapy ranged from $45 \%$ (after 4 weeks of treatment) to $73 \%$ (after 12 weeks of treatment) [2, 6]. When high frequency ultrasound therapy was used, the mean percentage reduction of the area of ulceration was noted from $57 \%$ (after 7 weeks of treatment) to $67 \%$ (after 12 weeks of treatment) $[4,5,7]$.

Our research shows that the use of ultrasound therapy with $1 \mathrm{MHz}$ and energy power density $0.5 \mathrm{~W} / \mathrm{cm}^{2}$ for 4 weeks results in an average reduction of $68 \%$ of the area of ulceration. This is a result comparable to that obtained by other authors.

The mean percentage reduction of ulceration area after shock wave application was 94\% (after 30 weeks of treatment - case report of refractory ulcer) to 100\% (after 6 weeks of treatment - case report). In addition, one of the authors described the treatment effects by the number of ulcers completely cured. This represented $36 \%$ of the treated group after 4-20 weeks of treatment. In this case, a $45 \%$ reduction of ulcer area was obtained [8-10].

We used venous leg ulcer treatment with radial shock wave therapy for 4 weeks, resulting in 38\% mean percentage reduction of ulceration area. This is a lower value than quoted in the available literature. The reason for this may be the type of shockwave we used. Radial shock wave is characterized by lower energy delivered to the tissues during the treatment compared to the focused shockwave. In addition, in the radial wave, the maximum energy is generated at the application site, while in the focal wave the maximum energy is below the surface of the ulcer. Moreover, we applied the shock wave for 4 weeks, while the cases described by us have a much longer treatment duration. Another difference is the number of patients. $94 \%$ and $100 \%$ reduction of ulcer area was reported in the case report. In cases where the number of patients is greater (11 persons), only $45 \%$ reduction of ulcer area was reported after 4 to 20 weeks. And this result is closer to ours.

The Gilman index was not assigned to any of the scientific articles cited by us. In our study Gilman index values at 4 weeks were significantly higher in the ultrasound therapy group than in other groups (shock wave therapy and control groups).

In the future, research is planned with long-term results (1 year after completion of therapy and observation of recurrence) rather than only the 4 weeks of therapy. Additionally, it is planned to expand the number of patient in comparative groups and perform analysis using parametric statistics.

\section{Conclusions}

Radial shock wave therapy, ultrasound therapy and standard care (gauze dressing saturated in $0.9 \%$ sodium chloride and elastic bandages) are effective methods of 
treating venous leg ulcers. The use of ultrasound therapy for the treatment of venous leg ulcers is more effective than the use of radial shock wave therapy. Standard care reduces the area of ulceration by only $16 \%$. The effect of treating venous leg ulcers with ultrasound therapy also reduces the cost of treatment. This happens because in a short time (4 weeks) the reduction of the area of ulceration is much greater than in other cases, using the methods described by us. This is important because of the ever-increasing number of patients affected by this condition.

\section{Acknowledgments}

This study was funded by statutory grant KNW-1101/N/5/0.

\section{Conflict of interest}

The authors declare no conflict of interest.

\section{References}

1. Nelson EA, Adderley U. Venous leg ulcers. Am Fam Phys 2017; 95: 662-3.

2. Escandon J, Vivas C, Perez R, et al. A prospective pilot study of ultrasound therapy effectiveness in refractory venous leg ulcers. Int Wound I 2012; 9: 570-8.

3. Gibbons GW, Orgil DP, Serena TE, et al. A prospective, randomised, controlled trial comparing the effects on noncontact, low-frequency ultrasound to standard care in healing venous leg ulcers. Ostomy Wound Manage 2015; 61: 16-29.

4. Olyale M, Rad FS, All Elahifar M, et al. High-frequency and noncontact low-frequency ultrasound therapy for venous leg ulcer treatment. Ostomy Wound Manage 2013; 59: 14-20.

5. Beheshti A, Shafigh Y, Parsa H, Zangivand A. Comparison of high frequency and MIST ultrasound therapy for the healing venous leg ulcers. Adv Clin Exp Med 2014; 23: 969-75.

6. White J, Ivnis N, Wilkes A, et al. Non-contact low frequency ultrasound therapy compared with UK standard of care for venous leg ulcers: a single-center, assessor-blinded, randomised controlled trial. Int Wound I 2016; 13: 833-42.

7. Taradaj J, Franek A, Błaszczak E, et al. Using physical modalities in the treatment of venous leg ulcers: a 14-years comparative clinical study. Wounds 2012; 24: 215-26.

8. Fioramonti P, Onesti MG, Finoi P, et al. Extracorporeal shock waves therapy for the treatment of venous ulcers in the lower limbs. Ann Ital Chir 2012; 83: 41-4.

9. Steiger M, Schmid JP, Bajrami S, Hunziker T. Extracorporeal shock wave therapy as a treatment of non-healing chronic leg ulcer. Hautarzt 2013; 64: 443-6.

10. Sagginii R, Figus A, Troccola A, et al. Extracorporeal shock waves therapy for management of chronic ulcers in the lower extremities. Ultrasound Med Biolog 2008; 34: 1261-71. 